\title{
Preparation of Multiphase Medical Device Materials with Ion Cross Section Polishing
}

\section{J.R. Heffelfinger}

Medtronic Inc., Energy and Component Center, 6700 Shingle Creek Parkway, Brooklyn Center, MN 55430

Preparation of medical device materials for characterization with optical or electron microscopy techniques is often complicated by the multiphase nature of the components. [1,2] Medical devices use a combination of polymer, metal and ceramic components. Each component has different mechanical properties which will affect sample preparation by conventional polishing techniques. In this study we use a JEOL 09010 Cross Section Polisher for the preparation of medical device materials.

The cross section polisher uses a beam of argon ions to mill away the surface of a sample. A mask is used to define the location of the milling. The energy and current of the argon beam can be tailored to the material type. Samples for milling can be prepared by traditional mounting and cross sectioning techniques, or by mechanically creating a shape that fits the sample holder. In our experience, milling takes between 4 to 6 hours depending on the sample. This will produce a polished surface that is up to $2 \mathrm{X} 2$ millimeters in size. As compared with focused ion beam (FIB) techniques, the cross section polisher poses advantages in the size of surface created in addition to size and cost of the equipment. [3]

Fig. 1a and 1b show SEM images of an aluminum/aluminum oxide composite material. This sample was prepared by fracturing. The metal and oxide components of the sample can be observed, however information about the metal/oxide interface and microstructure of the porosity is lost. Due to the brittle nature of the aluminum oxide, porosity of the material and the ductile nature of the aluminum, conventional cross sectioning methods tend to crush the microstructure of the sample.

Fig $2 \mathrm{a}$ and $2 \mathrm{~b}$ show SEM images of the same aluminum/aluminum oxide material that was prepared with the ion cross section polisher. The sample was milled for four hours with a $5 \mathrm{keV}$ accelerating voltage and approximately 100 micro amps of beam current. Milling produced a 1500 X 120 micrometer region of polished surface that was tapered in size from top to bottom. Pore structure of the sample showed clear channels and pores that extended into the sample. The geometry of the pores was maintained after polishing. Fine details of the metal/oxide interface could also be observed. Some polishing artifacts and grooves could be observed on the sample, especially near the bottom of the sample. The ion cross section polisher has been found to be useful in a number of medical device applications. Examples of other materials prepared by the ion polisher will be presented.

\section{References}

[1] D.A. Medlin, Abstracts of Microsc. Microanal. (2003)

[2] E.F. Schumacher, A.S. Teetsov, and R.M. Urban, Abstracts of Microsc. Microanal. (2004)

[3] M. Shibata, JEOL News, 39 No. 1 (2004) pp. 28-31. 

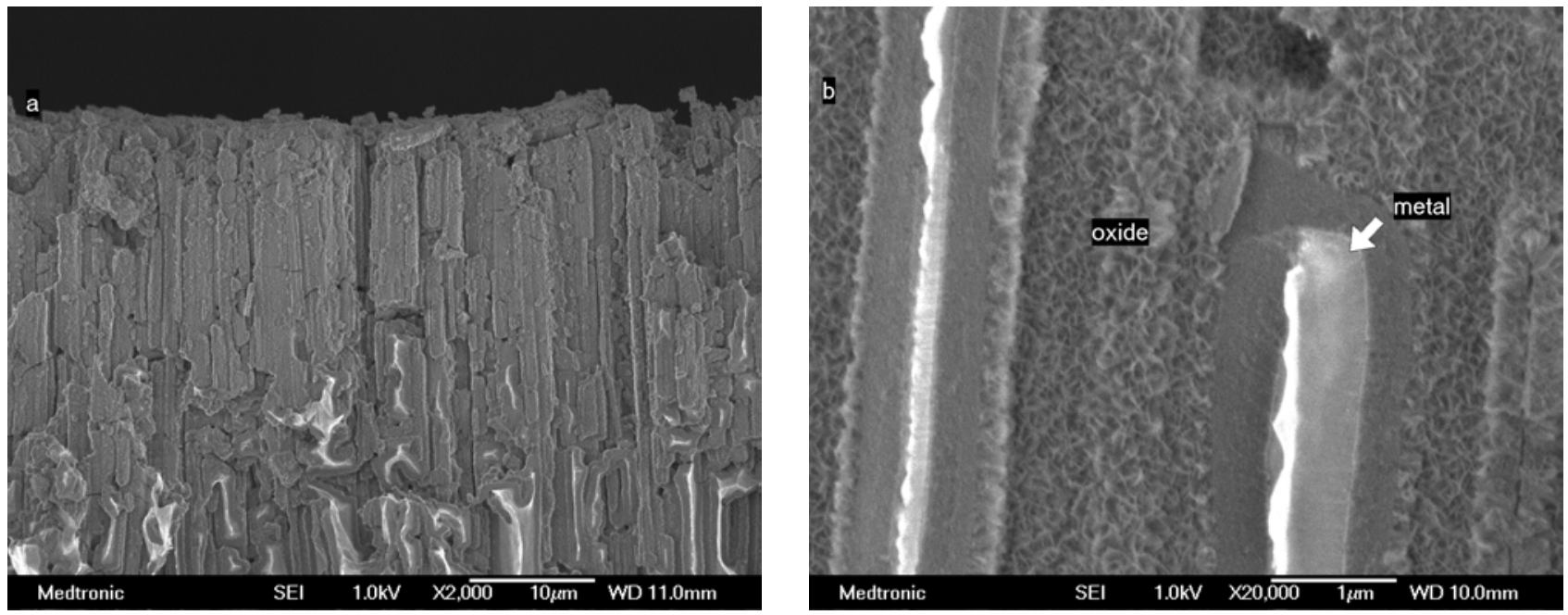

FIG. 1a and 1b. Secondary electron images of an aluminum/aluminum oxide composite. The brittle sample was prepared by fracturing the foil. In Fig. 1b, the ductile metallic region is observed to neck to a tear ridge.
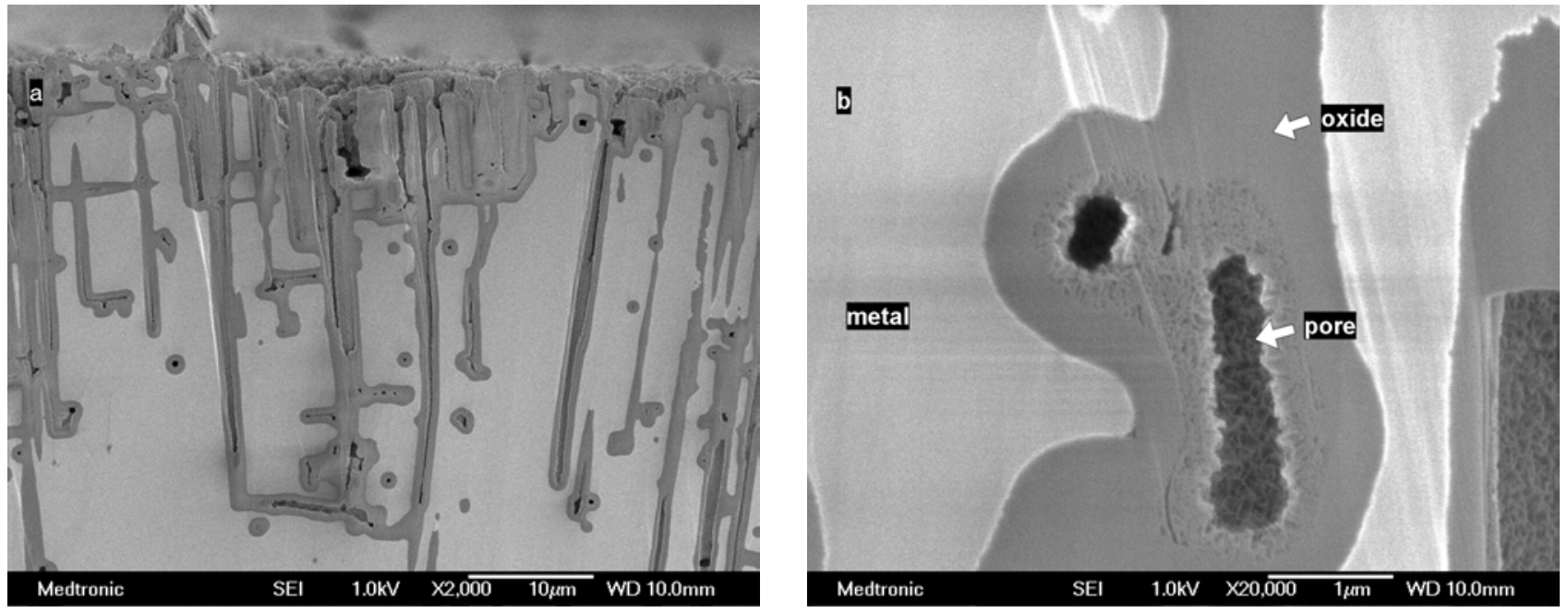

FIG. $2 \mathrm{a}$ and $2 \mathrm{~b}$. Secondary electron images of an aluminum/aluminum oxide composite. The brittle sample was prepared by ion cross section polishing. Fig 2 a shows the distribution and geometry of the oxide channels. Fig $2 b$, shows the interface of the metal and oxide regions. 